
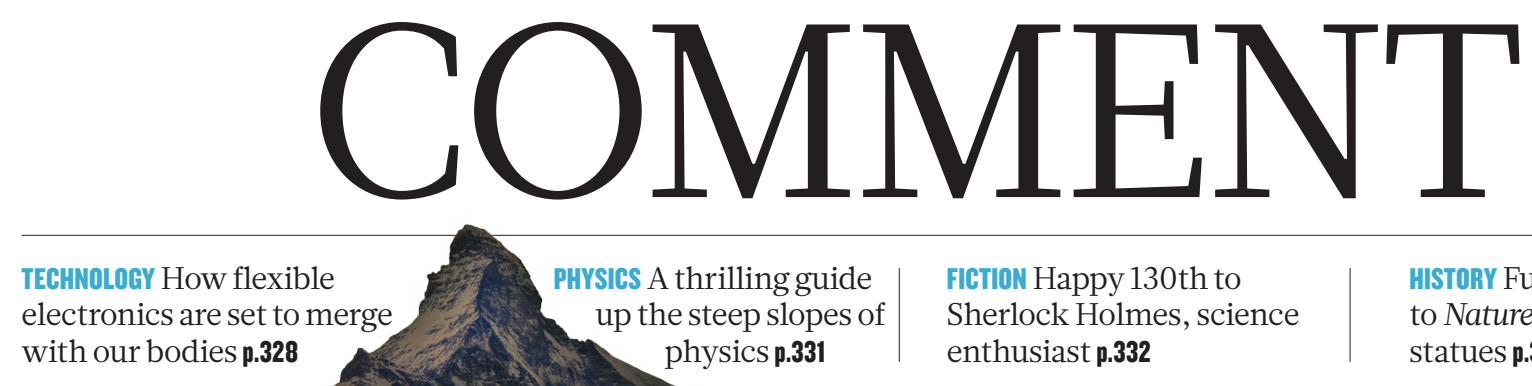

FICTION Happy 130th to

Sherlock Holmes, science enthusiast $\mathbf{p . 3 3 2}$
HISTORY Further responses to Nature's editorial on statues p.334

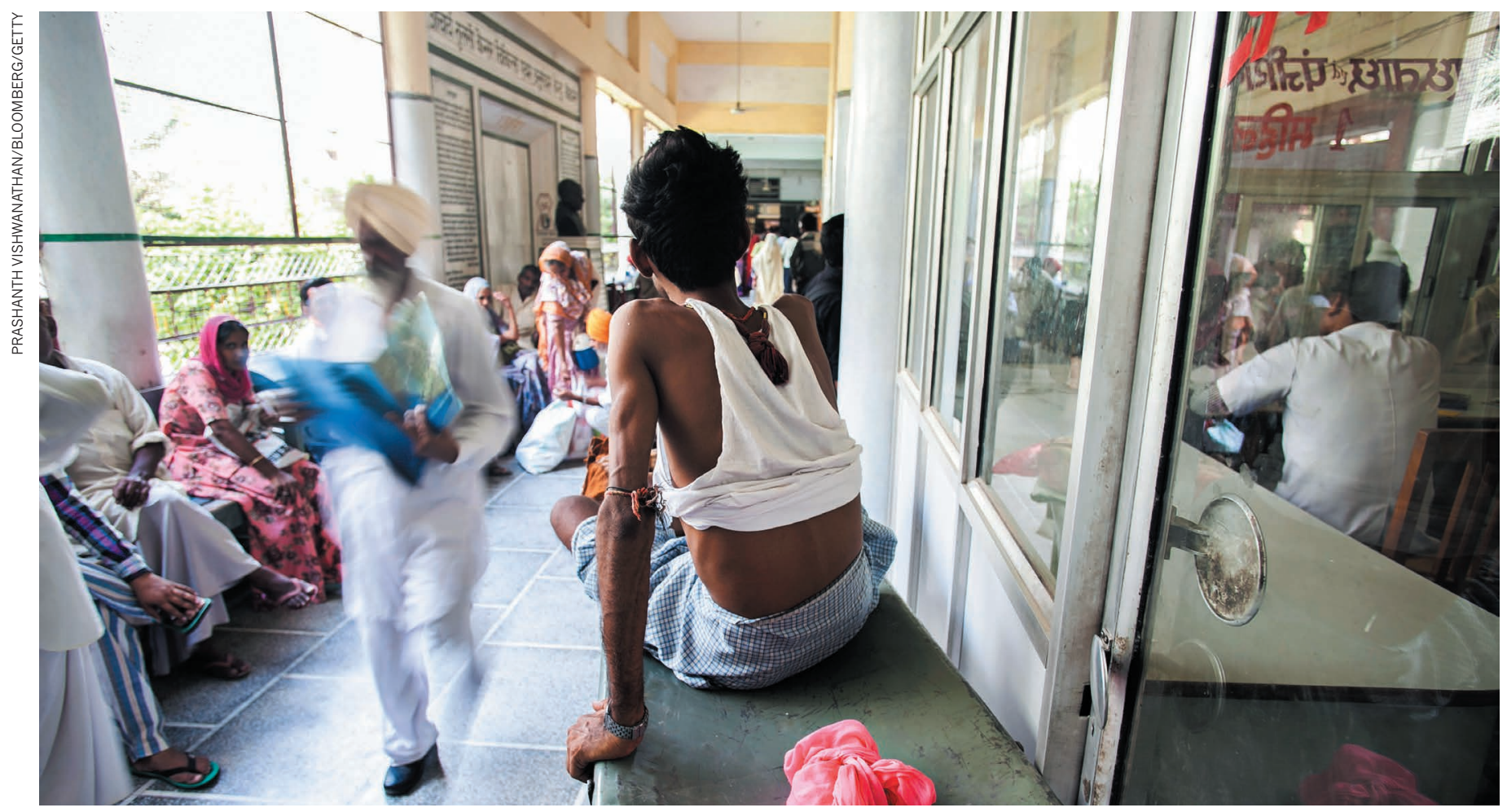

A patient awaiting treatment for cancer in an Indian hospital.

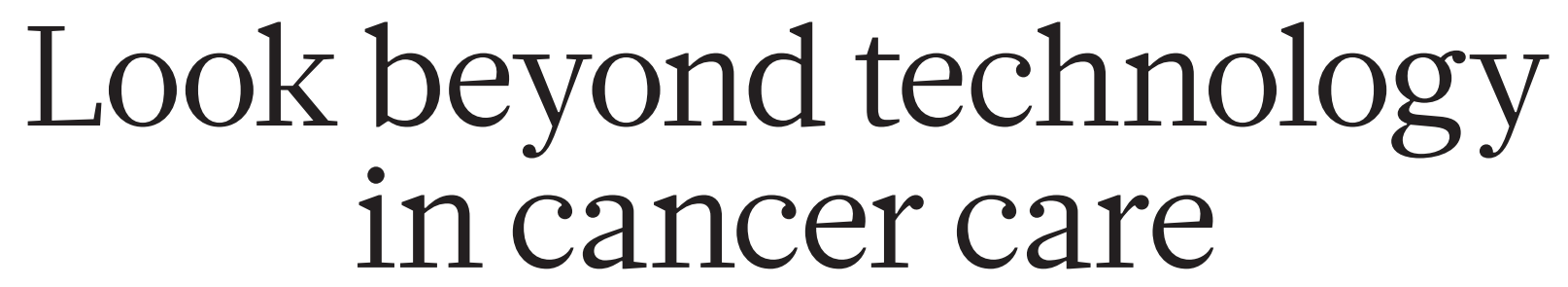

Treating cancer with the latest drugs and techniques is costly and will not improve survival globally, warn Richard Sullivan, C. S. Pramesh and Christopher M. Booth.

I n Nigeria, Malaysia, India and many other low- and middle-income coun-

tries, it is common to see hundreds of people queueing in the street to see a cancer doctor. It's also common in those regions to see people with curable cancer having chemotherapy, but not radiotherapy or surgery. In fact, $90 \%$ of people in low-income countries lack access to basic radiotherapy.

In wealthy countries, the push to develop new drugs, surgery and radiation techniques to treat cancer is at best unsustainable. Of 277 cancer-drug therapies for which clinical trials were published in 2011-15, only $15 \%$ identified treatments that led to meaningful improvements in patient survival or quality of life ${ }^{1}$. Indeed, studies reveal that the more expensive the drug, the less clinical benefit it seems to give ${ }^{2}$ (see 'A world of difference', panel a).

In middle- and low-income countries the technology-centric approach to cancer threatens to do more harm than good.

For the past 15 years, we have worked as clinical researchers in some 40 countries and conducted more than a dozen studies on national cancer-control planning. Our experiences - along with epidemiological and other data collected over 20 years indicate that the countries that rate relatively poorly on measures of cancer survival and mortality do so largely because of deficits at the political, economic and social level.

To improve the survival and well-being of the roughly 16 million people who have cancer worldwide, researchers, physicians, policymakers and patient organizations must focus on education, stigma, training and staffing to ensure that the right care 
$\checkmark$ is delivered to the right patient at the right time.

\section{A GROWING PROBLEM}

Cancer is on the rise. Ten years ago, 12.7 million people worldwide were living with the disease, with an economic impact of nearly US $\$ 290$ billion. By 2030, 21.7 million people are expected to be affected, at an anticipated cost of $\$ 458$ billion - largely because of a growing and ageing population as well as lifestyle changes. But the numbers vary dramatically across countries.

Among European nations, there are huge differences in mortality and morbidity for every type of the disease, according to the EUROCARE-5 database ${ }^{3}$ (see 'A world of difference', panel b). For example, in 2014 there was a $14.5 \%$ difference in survival rates for patients with breast cancer living in Denmark (one of the best-performing countries) and those in Lithuania (the worst). For rectal cancer, survival rates differed from country to country by as much as $32 \%$.

Likewise, in Asia the proportion of patients who died one year after being diagnosed with solid tumours, such as breast or colorectal cancer, ranged from $12 \%$ (in Malaysia) to $45 \%$ (in Myanmar), according to a 2012 study $^{4}$. Meanwhile, the proportion of patients facing destitution after paying for treatment ranged from one-quarter in Thailand to two-thirds in Vietnam ${ }^{4}$ (see 'A world of difference', panel c).

Many hospitals in emerging economies and most in low-income countries lack the basic infrastructure and personnel needed to treat diverse cancers. The Tata Memorial Centre in Mumbai, for instance (where C.S.P. works), is the oldest and largest cancer-treatment and research centre in India. It has 164 senior faculty members, who see roughly 40,000 patients each year. Compare that to the MD Anderson Cancer Center in Houston, Texas, which sees 33,000 patients per year but has more than 11 times as many senior faculty members $(1,834)$.

Similarly, in sub-Saharan Africa, only 16 countries have access to basic pathology services - trained staff with the equipment needed to make a diagnosis of cancer. Throughout much of Africa, there is on average one pathologist for every 2.3 million people. In high-income countries, there is typically one pathologist for every 15,000 to 20,000 people .

Even in well-resourced urban areas, a lack of guidelines and auditing undermines the effectiveness of many clinical labs. In 2011, only 5\% of the 954 pathology labs in Kampala, Uganda, met the minimum tissue-handling and reporting standards defined by the World Health Organization (WHO) Regional Office for Africa $^{6}$. In our experience, poorly trained staff often make incorrect diagnoses, or produce reports without analysing tissue.

Another barrier to improving outcomes is that patients do not see doctors early enough in their disease. The five-year survival rate for breast cancer is only $68.4 \%$ in Tunisia. This is in part because, in many low- and middle-income countries, women with cancer can be stigmatized by their communities. Many must obtain permission from their husbands to see a doctor, and are fearful that a cancer diagnosis will lead to divorce.

"Throughout
much of
Africa, there is
on average one
pathologist
for every
2.3 million
people."

Everywhere, a lack of education and awareness, affordability and availability of treatment are the main factors preventing patients from being diagnosed early enough $^{7,8}$. Given all this, it is alarming that many low- and middle-income countries are devoting more of their meagre cancer-care budgets to technology, especially through the private sector.

\section{CONSIDER THE COST}

The past decade has witnessed an explosion of targeted and immunotherapeutic drugs for cancer. The number of new technologies in surgery, particularly in robotics, has also risen exponentially. New radiation techniques such as tomotherapy (a form of computed tomography in which radiation is targeted at specific slices of the body) and proton-beam therapy are also being rolled out each year.

Many emerging economies are now investing in these high-tech interventions ${ }^{9}$, even though they lack the purchasing and negotiating powers of high-income countries and do not have systems to determine the cost-effectiveness of what they're buying.

The drug bevacizumab, for example, costs between $\$ 4,000$ and $\$ 5,000$ per month in the United States compared to the drug tamoxifen, which costs approximately $\$ 50$ per month. The former is now considered a standard treatment in India for patients with metastatic colon cancer, despite trials showing that it improves median survival by only 6 weeks ${ }^{10}$. Meanwhile, in many parts of India, there aren't enough pathologists to test a woman's breast cancer for the oestrogen receptor. Such information would enable many thousands of women to receive tamoxifen, which increases the cure rate of breast cancer by $10 \%$ (ref. 11).

Furthermore, despite considerable uncertainty about the cost-effectiveness of protonbeam therapy, there are plans to install at least 18 such machines across Brazil, Russia, India, China and South Africa. Each machine costs around $\$ 140$ million $^{12}$. These same countries currently have an average deficit of around $60 \%$ in both human resources and equipment for basic radiotherapy, which is much more effective in increasing cure rates and relieving suffering ${ }^{13}$.

Less than $5 \%$ of patients in low-income countries have access to safe, affordable and timely cancer surgery; for middle-income countries the situation is only marginally better, at around 22\% (ref. 14). Yet these same countries are spending hundreds of millions of US dollars each year on immunotherapeutic drugs.

In short, in most emerging economies, there is a chronic under-use of therapies that can save lives (such as cervical-cancer screening, basic surgery and radiotherapy) and a chronic over-use of interventions that, at huge expense to the patient, provide no meaningful benefit.

\section{THREE MAJOR SHIFTS}

To better balance innovation in cancer drugs and therapies with the requisite social, economic and structural investments requires three major shifts.

Change global mindsets. Media hype fuels the perception that new must mean better $^{15}$. The complexity of the disease probably also makes it easy for the various players of the medical-industrial complex to persuade policymakers to prioritize hightech solutions.

Cancer-advocacy bodies, researchfunding organizations and patient groups must stop advocating access to expensive (and often low-value) technologies, especially in low- and middle-income countries. In the past year, hundreds of policy briefings produced by groups such as these have asked governments for investments.

Also, more scientific rigour, media scrutiny and public debate globally could make it harder for the cancer community (including researchers, physicians and patient groups) to celebrate marginal wins and endorse policy focused on technological innovation ${ }^{15}$.

In parallel, the bar must be raised, such that health-insurance companies, governments and other payers fund only those interventions that have a meaningful impact on patients' lives. This means basing decisions about which technologies to incorporate into routine clinical care, and how much to invest in them, on survival and quality of life. Surrogate endpoints, such as lack of tumour growth and radiological and biomarker responses, have increasingly been used in recent years, even though, in many cases, investigators have not been able to correlate them with outcomes that are meaningful to patients ${ }^{16,17}$.

Fund human capital and social development. Spending needs to be redistributed more evenly between people, basic technologies and the delivery of safe, affordable cancer care. Governments and development organizations, such as the World Bank and the 
* WHO, need to prioritize education and the employment of more cancer-specific healthcare workforces. They also need to address the basic social factors that determine whether patients are seen and diagnosed early enough.

This multi-pronged approach has proved successful in other contexts. The international effort to combat HIV/AIDS has been as much about social engineering as developing drugs and vaccines. Researchers working on antiretrovirals have actively supported and engaged with those in community development, education and the design of effective pathways for care.

For cancer, some collaborations have already yielded important advances. A partnership involving Moi University in Eldoret, Kenya, Indiana University in Indianapolis and other high-income cancer centres has helped more than 4,500 Kenyans obtain highquality cancer care over the past five years largely through sharing models of care and facilitating the training of surgeons and other carers $^{18}$. And the Indian government's support for the transfer of cost-effective, Indianmade radiation equipment to countries such as Vietnam and Mongolia, among others, has provided numerous underserved communities with access to radiation therapy.

Governments must not insist on replicating models established in high-income countries. In place of medical oncologists, for example, surgeons could be trained to deliver basic low-risk chemotherapy, and nurses taught to deliver palliative care. Expanding the skill sets of general doctors and surgeons, and training more pathologists, would also help.

Likewise, investing more in cancer prevention and public health would pay huge dividends. It has been estimated that, across Europe, driving tobacco usage down to a level at which less than $5 \%$ of the population uses it would plough some $€ 10$ billion (US $\$ 12$ billion) back into economies each year by preventing premature deaths due to tobacco-related cancers.

Implement standards and systems for accountability. In our experience, pharmaceutical companies are beginning to recognize that without improvements to systems and processes, their sales will stagnate, particularly in emerging and low-income economies. But expensive cancer medicines are still a major drain on resources. National health-insurance systems, such as those in India and Mexico, need to do a much better job of spending government money only on effective care. Developing country-specific management guidelines, as has been done by the National Cancer Grid of India, and linking government insurance reimbursements to adherence to these could further encourage providers to deliver evidencebased care. Indeed, the misuse of technology in cancer care for profit is a major issue

\section{A WORLD OF DIFFERENCE}

a

In Europe, higher levels of spending on prostate-cancer patients are associated with higher fatality rates.

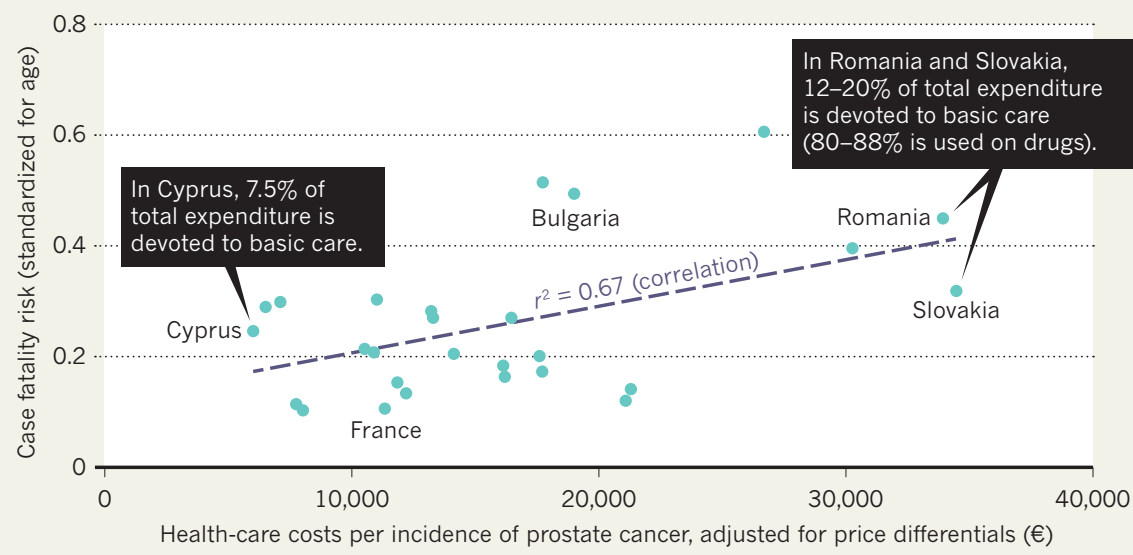

Mortality for every type of cancer differs greatly between European nations (b), largely because of differences in investment in basic care. In some southeast Asian countries, the likelihood of patients dying or experiencing financial catastrophe is similarly variable (c).

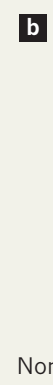

b

$$
\begin{array}{r}
\text { Testis } \\
\text { Thyroid } \\
\text { Skin melanoma } \\
\text { Prostate }- \\
\text { Hodgkin's lymphoma } \\
\text { Breast (women) } \\
\text { Corpus uteri } \\
\text { Urinary bladder } \\
\text { SBLL/CLL* } \\
\text { Cervix uteri }- \\
\text { Kidney }
\end{array}-
$$

- Outlier countries

Non-Hodgkin's lymphoma

Larynx

Colon

Colon-rectum

Rectum

Head and neck

Ovary

Plasma cell

Stomach

Brain

Gall bladder

$\mathrm{AML}^{*}$

Lung

Oesophagus

Liver

Pancreas

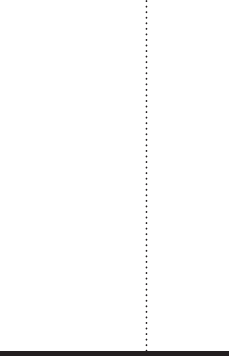

Worst-performing country

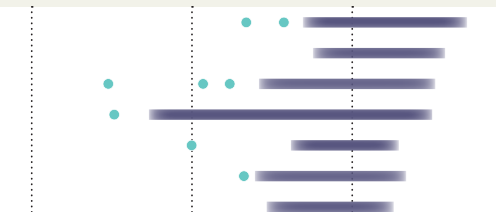

.
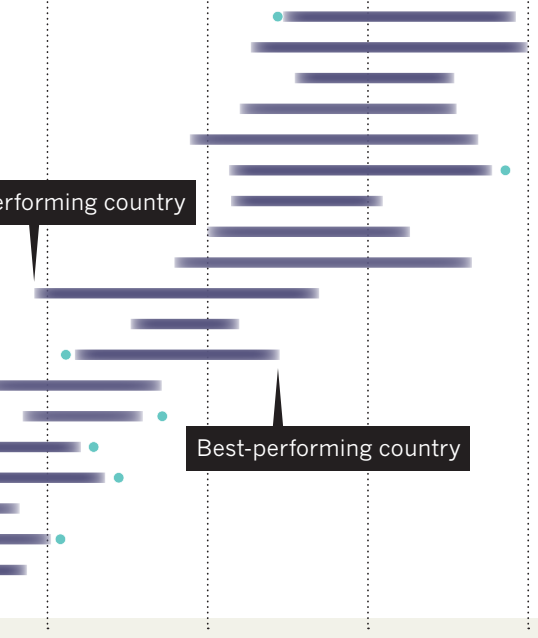

\section{y}
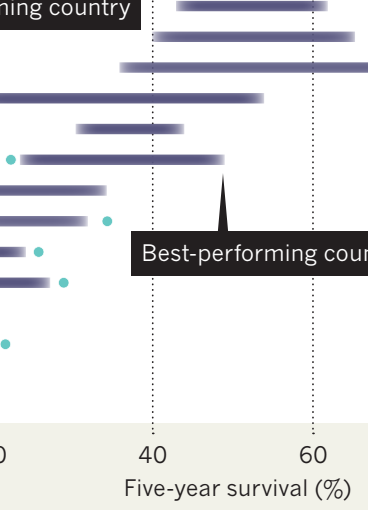

c
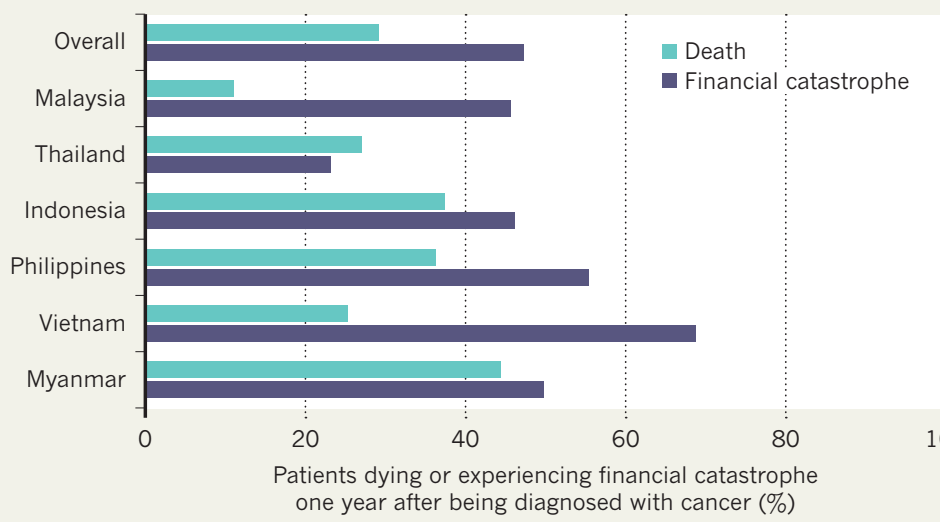

*SBLL/CLL, small B-cell lymphocytic lymphoma or B-cell chronic lymphocytic leukaemia; AML, acute myeloid leukaemia. 
$\checkmark$ in many countries where health care is unregulated $^{19}$.

Systems of accreditation for cancer centres (public and private) could also help to ensure that institutions offer interventions only after demonstrating competence and achieving certain scores from patient feedback and peer review. They may also encourage the establishment of specialist centres. Data from the past 25 years have shown that cancer patients do much better if the surgeon treating them has operated on many others with the same condition as part of a multidisciplinary team ${ }^{20}$.

Cancer 'moonshots' may improve individual outcomes in high-income countries with strong governance, but they will not solve the rising economic and social burden of cancer globally. What we need are 'earthshots' that focus on building infrastructure and delivering affordable, equitable and effective care.

Richard Sullivan is a professor of cancer and global health at King's College London, and director of the King's Institute of Cancer Policy, London, UK. C. S. Pramesh is a professor of thoracic surgery at the Tata Memorial Centre, Mumbai, India, and convener of the National Cancer Grid of India. Christopher M. Booth is a professor and research chair in population cancer care at Queen's University Cancer Research Institute, Kingston, Canada. e-mail:richard.sullivan@kcl.ac.uk

1. Del Paggio, J. C. et al. Ann. Oncol. 28, 157-162 (2017)

2. Del Paggio, J. C. et al. Lancet Oncol. 18, 887-894 (2017).

3. De Angelis, R. et al. Lancet Oncol. 15, 23-34 (2014).

4. Kimman, M. et al. BMC Med. 13, 190 (2015).

5. Nelson, A. M., Milner, D. A., Rebbeck, T. R. \& Iliyasu, Y. J. Clin. Oncol. 34, 20-26 (2016).

6. Elbireer, A. M. et al. PLoS ONE 8, e64661 (2013)

7. MacLeod, U., Mitchell, E. D., Burgess, C. Macdonald, S. \& Ramirez, A. J. Br. J. Cancer 101 (Suppl. 2), S92-S101 (2009).

8. Donkor, A. et al. Arch. Med. 8, 2 (2015)

9. Saltz, L. B. et al. J. Clin. Oncol. 26, 2013-2019 (2008).

10 Pramesh, C. S. et al. Lancet Oncol. 15 e223-e233 (2014)

11. Early Breast Cancer Trialists' Collaborative Group (EBCTCG) Lancet 378, 771-784 (2011).

12. Verma, V., Mishra, M. V. \& Mehta, M. P. Cancer 122, 1483-1501 (2016).

13. Datta, N. R., Samiei, M. \& Bodis, S. Int. J. Radiat. Oncol. Biol. Phys. 89, 448-457 (2014).

14. Sullivan, R. et al. Lancet Oncol. 16, 1193-1224 (2015).

15. Sullivan, R., Behncke, I. \& Purushotham, A. EMBO Rep. 11, 572-578 (2010)

16. Kay, A., Higgins, J., Day, A. G., Meyer, R. M. \& Booth, C. M. Ann. Oncol. 23, 1646-1651 (2012).

17. Booth, C. M. \& Eisenhauer, E. A. J. Clin. Oncol. 30, 1030-1033 (2012)

18. Strother, R. M. et al. J. Cancer Policy 1, e42-e48 (2013).

19.Pramesh, C. S. et al. Lancet Oncol. 15, 223-233 (2014).

20. Birkmeyer, J. D. et al. N. Engl. J. Med. 349 2117-2127 (2003).

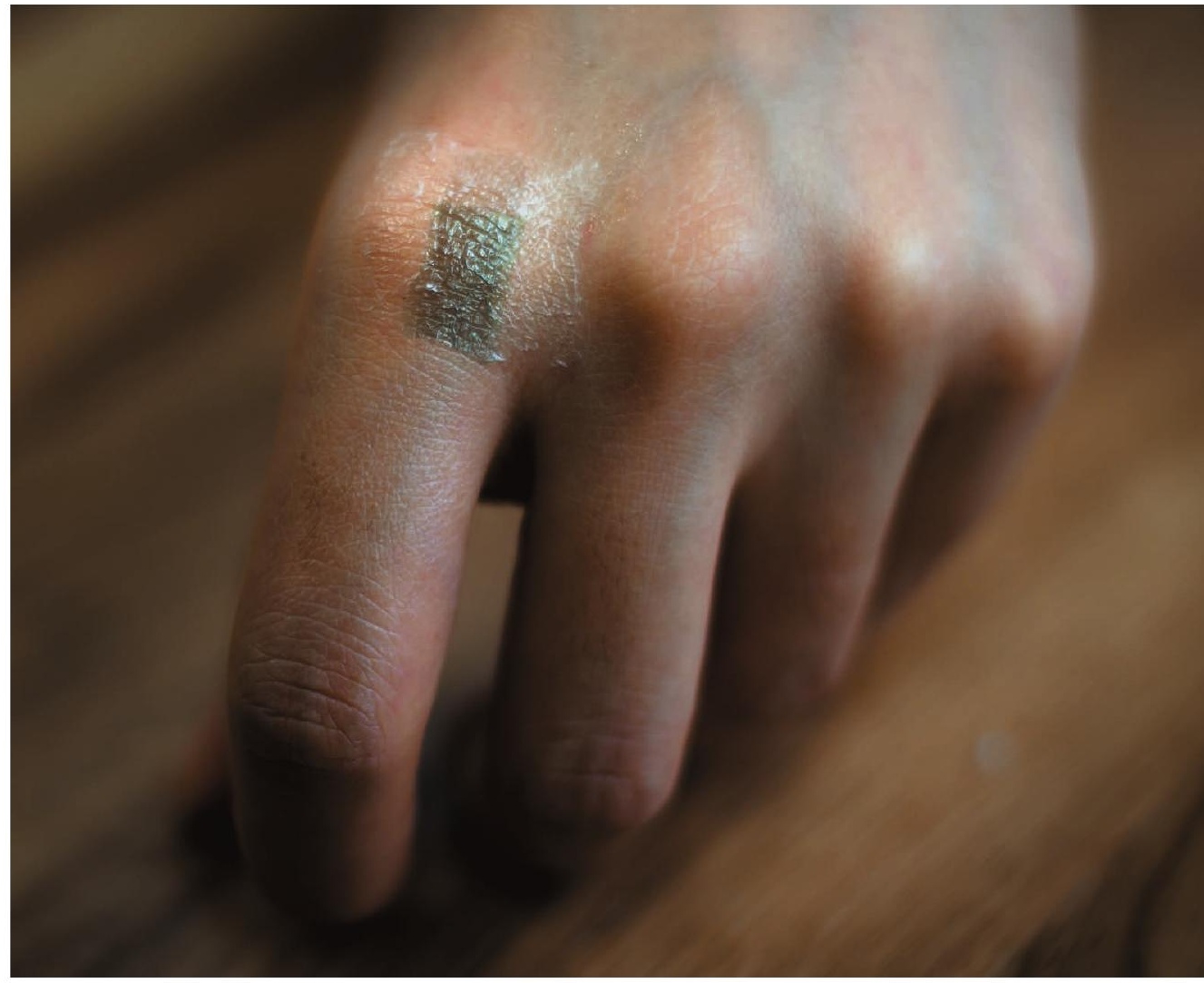

Unobtrusive 'elastronic' transistors can behave like skin and stretch without tearing.

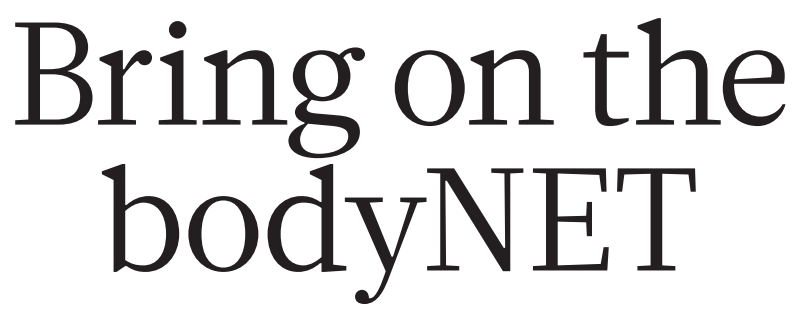

\section{Stretchable sensors, circuits and batteries are about to change our relationships with electronics and each other, explain Bryant Chu and colleagues.}

$\mathrm{E}$ lectronics are set to merge with our bodies to extend our perceptions. Smartphones and watches will give way to the bodyNET ${ }^{1}$ : a network of sensors, screens and smart devices woven into our clothing, worn on our skin and implanted in our bodies (see 'Superhuman powers'). A pregnant woman might wear tiny biometric sensors to monitor her baby's heartbeat, displayed on a film attached to her skin. She could transmit its kicks to the father wirelessly, so that he can experience the vibrations recreated by 'haptics' - interfaces that provide tactile feedback - on his stomach.

The bodyNET is not yet complete, and labs around the world are developing its components. The core technology is electronics that stretch - elastronics - made from soft plastic circuits thinner than paper that can deform without tearing, biodegrade and even heal themselves (see go.nature.com/2vtutzz) Elastronic sensors respond to touch, pres sure, temperature, humidity and light, as well as to chemical and biological signals ${ }^{2-10}$

There is much still to do. Researchers must improve the technical performance of elastronic materials, design innovative architectures for stretchable circuits and drive down costs through mass production. There are also social and cultural concerns. These include widespread fears of merging technology intimately with the body, as well as anxieties about privacy and data security.

Yet we are optimistic that the benefits of bodyNETs will outweigh the challenges. These extensions of ourselves will allow us to sense and communicate with others and our surroundings in new and sophisticated ways, beyond our existing five senses. Being able to see how a patient is feeling in real time, or 